\title{
Identification of stable endogenous control genes for transcriptional profiling of photon, proton and carbon-ion irradiated cells
}

Geeta D Sharungbam ${ }^{1,2}$, Christian Schwager ${ }^{1,2}$, Sara Chiblak ${ }^{1,2}$, Stephan Brons², Lynn Hlatky ${ }^{3}$, Thomas Haberer ${ }^{2}$, Jürgen Debus ${ }^{2}$ and Amir Abdollahi ${ }^{1,2,3^{*}}$

\begin{abstract}
Background: Quantitative analysis of transcriptional regulation of genes is a prerequisite for a better understanding of the molecular mechanisms of action of different radiation qualities such as photon, proton or carbon ion irradiation. Microarrays and real-time quantitative RT-PCR (qRT-PCR) are considered the two cornerstones of gene expression analysis. In interpreting these results it is critical to normalize the expression levels of the target genes by that of appropriately selected endogenous control genes (ECGs) or housekeeping genes. We sought to systematically investigate common ECG candidates for their stability after different radiation modalities in different human cell lines by qRT-PCR. We aimed to identify the most robust set of ECGs or housekeeping genes for transcriptional analysis in irradiation studies.

Methods: We tested the expression stability of 32 ECGs in three human cancer cell lines. The epidermoid carcinoma cells (A431), the non small cell lung carcinoma cells (A549) and the pancreatic adenocarincoma cells (BxPC3) were irradiated with photon, proton and carbon ions. Expression Heat maps, clustering and statistic algorithms were employed using SUMO software package. The expression stability was evaluated by computing: mean, standard deviation, ANOVA, coefficient of variation and the stability measure $(M)$ given by the geNorm algorithm.
\end{abstract}

Results: Expression analysis revealed significant cell type specific regulation of 18 out of 32 ECGs $(p<0.05)$. A549 and A431 cells shared a similar pattern of ECG expression as the function of different radiation qualities as compared to BxPC3. Of note, the ribosomal protein $18 \mathrm{~S}$, one of the most frequently used ECG, was differentially regulated as the function of different radiation qualities $(p \leq 0.01)$. A comprehensive search for the most stable ECGs using the geNorm algorithm identified 3 ECGs for A431 and BxPC3 to be sufficient for normalization. In contrast, 6 ECGs were required to properly normalize expression data in the more variable A549 cells. Considering both variables tested, i.e. cell type and radiation qualities, 5 genes-- RPLPO, UBC, PPIA, TBP and PSMC4- were identified as the consensus set of stable ECGs.

Conclusions: Caution is warranted when selecting the internal control gene for the qRT-PCR gene expression studies. Here, we provide a template of stable ECGs for investigation of radiation induced gene expression.

Keywords: Endogenous control genes, Internal control genes, qRT-PCR, Photon, Proton, Carbon-ion, Tumour cells, A431, A549 and BxPC3

\footnotetext{
* Correspondence: a.amir@dkfz.de

${ }^{1}$ Molecular RadioOncology [E210], National Center for Tumor Disease (NCT),

German Cancer Research Center (DKFZ), Im Neuenheimer Feld 460, 69120,

Heidelberg, Germany

${ }^{2}$ Heidelberg lon Therapy Center (HIT), Heidelberg Institute of Radiation

Oncology (HIRO), Department of Radiation Oncology, University of

Heidelberg Medical School, Im Neuenheimer Feld 450, 69120, Heidelberg,

Germany

Full list of author information is available at the end of the article
} 


\section{Background}

In addition to direct, e.g. DNA damaging effect, system level cellular responses to ionizing radiation are attributed to the initiation of intracellular signals and subsequent differential regulation of genes/pathways governing various cellular processes [1]. Therefore, detecting differential regulation of genes is critical for a better understanding of radiation-induced molecular effects. Transcriptional perturbation after cell exposure to different radiation qualities is investigated to unravel the systems biology of cellular response underlying, normal tissue toxicity, carcinogenesis, or anti-cancer effects of irradiation [1-3]. Therefore, these studies have ramification for a broad spectrum of basic and applied sciences ranging from effects of space radiation to carcinogenesis to cancer therapy.

In contrast to conventional photon irradiation the molecular effects of proton or heavier ions (e.g. carbon ions) are less explored yet. However, emerging data indicate molecular differences in transcriptional response of cells to particles as compared to photon irradiation $[4,5]$.

One reliable and highly sensitive tool that allows rapid and accurate results in gene expression analysis is the qRT-PCR [6,7]. As in any gene expression analysis, selection of a valid normalization or endogenous control to correct for differences in RNA sampling is critical to avoid misinterpretation of results. Inter-sample variation due to sample collection, RNA preparation and quality, inherent sample differences, pipetting errors, different efficacies of the radiation qualities and reverse transcription efficiency are common sources of variability. The ideal endogenous control should have a constant expression level under different experimental conditions and be sufficiently abundant across different samples and cell lines. Although any gene that is stably expressed under a defined experimental condition can be used for normalization, the selection is most commonly made from the constitutively expressed ECGs.

However, the expression levels of the commonly used ECGs may not only vary in different cell lines but also under different experimental treatments or pathological states [8-25]. This necessitates the selection of ECGs which are appropriate for each experimental system. Although, there has been systematic selection of ECGs for various experimental systems, such selection has not been conducted so far for studying the effects of different radiation qualities.

Here, we investigate the expression stability of 32 commonly used ECGs in three human cancer cell lines irradiated with photon, proton and carbon ions. Differential regulation of ECGs was found as the function of both variables, radiation quality and cell type, respectively. Reliable internal control genes for individual cell lines were identified such as PGK1, RPL37A and PSMC4 for A431; RPLPO, UBC, GAPDH, MT-ATP6, CASC3 and
PES1 for A549; and RPL37A, RPLPO and CASC3 for BxPC3. A systematic analysis further revealed 5 stable genes among the 32 candidate ECGs tested to normalize gene expression data generated in different cells and after various radiation qualities.

\section{Results}

\section{Expression of the 32 ECGs}

In this study, 32 ECGs (Additional file 1) were evaluated to identify the most suitable reference genes for gene expression profiling of irradiated cell lines. This collection of genes constitutes frequently used ECGs which were selected based on their relative high abundance and constitutive expression determined by literature search and/or whole genome microarray data. The three prototypic tumour cell lines-- A431, A549 and BxPC3 used in this study are among most commonly investigated model cell lines for each tumor entity. They were irradiated with photon, proton and carbon ions. After total-RNA isolation and quality control using lab-on-chip bioanalyzer, qRTPCR was performed using Taqman primer and probes.

\section{$C T$-values and ECG regulation}

To get a better overview of the CT-values among all the cell lines, the CT-range along with the minimum and maximum CT-values were listed in Table 1 . The variation of CT-values ranged from 8.97 in A431 to 28.58 in A549. The 8.97 CT-value corresponded to $18 \mathrm{~S}$ indicating its high abundance in the samples, whereas, the CTvalue 28.58 corresponded to GADD45A indicating a moderate abundance. Moreover, RPL37A, GAPDH and $R P L P O$ exhibited small CT-range indicating less variation in expression whereas the large CT-range of $A C T B$, GADD45A, IPO8 showed large variations in their expression.

The CT-range and coefficient of variation for each cell line were listed in additional file 2 . Within each individual cell line irradiated with photon, proton and carbon ion, all the ECGs in BxPC3 except for CDKN1A, 18S, POLR2A, PES1 and HMBS with CT-range 1.18, 0.82, $0.80,0.70$ and 0.61 , respectively, showed the smallest CT-range, while in A549 all the ECGs except PES1, $A C T B$ and RPS17 showed the largest CT-range (Figure 1A). In other words, as a function of different radiation qualities in BxPC3, the expression of the ECGs was considerably stable, while there was more variation in A549. Moreover, A431 and A549 shared similar expression pattern of the ECGs. The coefficient of variation plotted in Figure 1B also reflected the similar regulation of the 32 ECGs in the three cell lines.

\section{ECG regulation using heat map}

The expression of the 32 candidate ECGs across the samples were also visualized in a Heat map (Figure 2). 
Table 1 Cycle threshold (CT) values and coefficient of variation (CV) of 32 endogenous control genes across the samples of the cell lines

\begin{tabular}{llllll}
\hline Gene Symbol & CT Range & CT Min. & CT Max. & Mean CT \pm SEM & CV(\%) \\
\hline RPL37A & 1.13 & 17.95 & 19.08 & $18.44 \pm 0.29$ & 1.6 \\
HMBS & 1.39 & 23.96 & 25.35 & $24.61 \pm 0.41$ & 1.7 \\
CASC3 & 1.22 & 22.79 & 24.01 & $23.45 \pm 0.42$ & 1.83 \\
RPLPO & 1.36 & 17.22 & 18.58 & $17.83 \pm 0.34$ & 1.91 \\
PSMC4 & 1.56 & 21.04 & 22.6 & $21.90 \pm 0.46$ & 2.12 \\
ABL & 1.92 & 22.95 & 24.87 & $23.71 \pm 0.51$ & 2.15 \\
UBC & 1.62 & 19.22 & 20.85 & $19.81 \pm 0.43$ & 2.19 \\
GAPDH & 1.25 & 16.01 & 17.25 & $16.53 \pm 0.37$ & 2.27 \\
POLR2A & 2.03 & 23.5 & 25.53 & $24.19 \pm 0.56$ & 2.35 \\
MT-ATP6 & 1.39 & 15.18 & 16.57 & $15.66 \pm 0.38$ & 2.46 \\
PES1 & 1.97 & 21.04 & 23.02 & $22.12 \pm 0.55$ & 2.49 \\
TBP & 2.19 & 23.88 & 26.08 & $24.47 \pm 0.64$ & 2.62 \\
GUSB & 1.7 & 22.28 & 23.98 & $23.30 \pm 0.63$ & 2.71 \\
EIF2B1 & 2.53 & 23.593 & 26.12 & $24.11 \pm 0.70$ & 2.91 \\
RPS17 & 1.6 & 17.58 & 19.18 & $18.14 \pm 0.53$ & 2.92 \\
RPL30 & 1.89 & 17.24 & 19.14 & $18.05 \pm 0.53$ & 2.96 \\
POP4 & 1.17 & 22.73 & 23.91 & $23.64 \pm 0.72$ & 3.05 \\
PPIA & 2.15 & 17.43 & 19.58 & $18.08 \pm 0.55$ & 3.07 \\
PUM1 & 2.88 & 22.15 & 25.03 & $23.10 \pm 0.71$ & 3.08 \\
HPRT1 & 2.73 & 21.18 & 23.91 & $22.08 \pm 0.68$ & 3.1 \\
CDKN1B & 2.08 & 22.8 & 24.98 & $23.98 \pm 0.81$ & 3.41 \\
PGK1 & 2.25 & 18.17 & 20.42 & $19.35 \pm 0.69$ & 3.61 \\
MRPL19 & 3.09 & 22.73 & 25.82 & $24.01 \pm 0.95$ & 3.96 \\
CDKN1A & 2.6 & 21.3 & 23.99 & $22.98 \pm 0.95$ & 4.17 \\
ACTB & 5.65 & 16.1 & 21.75 & $17.80 \pm 0.75$ & 4.23 \\
ELF1 & 3.59 & 21.81 & 25.39 & $22.76 \pm 1.00$ & 4.4 \\
IPO8 & 4.26 & 23.68 & 27.93 & $24.80 \pm 1.13$ & 4.57 \\
GADD45A & 4.2 & 24.37 & 28.57 & $26.29 \pm 1.37$ & 4.97 \\
YWHAZ & 3.56 & 22.17 & 25.73 & $23.96 \pm 1.26$ & 5.27 \\
TFRC & 4.26 & 21.15 & 25.41 & $22.13 \pm 1.18$ & 5.37 \\
B2M & 3.76 & 17.99 & 21.75 & $19.33 \pm 1.21$ & 6.29 \\
THE 9 3.95 & 8.79 & 11.95 & $10.78 \pm 1.02$ & 9.5 \\
\hline
\end{tabular}

The genes are sorted by the coefficient of variation increasing from top to bottom.

Four replicates were used for each cell line. Cell lines were irradiated with photon, proton and carbon ions. Non-irradiated samples served as control. Standard error of the mean (SEM).

Direct clustering of the ECGs expression showed that the expression profile of A431 and A549 were more similar as compared to $\mathrm{BxPC} 3$. BxPC3 showed least variation whereas A549 showed maximum variation in expression among the samples as well as among the ECGs indicating a cell type specific expression of ECGs. As observed in Table 1, the Heat map also revealed low variation of RPL37A, RPLPO and GAPDH expression, while $A C T B, I P O 8$ and GADD45A depicted high variation in expression. The remaining ECGs were differentially regulated. For example, $B 2 M$ and $E L F 1$ were up regulated in BxPC3 and $\mathrm{A} 431$ but down regulated in A549. On the other hand, MRPL19 and PES1 were down regulated in BxPC3 but up regulated in A431 and A549.

In Figure 3, we displayed a Heat map generated from a one way ANOVA analysis at $p<0.05$ between the three cell lines. It revealed 18 differentially regulated ECGs-ABL, CDKN1A, PSMC4, EIF2B1, GAPDH, PPIA, TBP, RPS17, UBC, B2M, ELF1, PUM, GADD45A, ACTB, MRPL19, YWHAZ, CASC3 and PES1. These could be cell type specific regulations as their radiation quality variation was minimal. Among them, the first 13 except for PSMC4 were up regulated in $\mathrm{BxPC} 3$ and the remaining down regulated. In A431 and A549, 12 of them were down regulated and 6 were up regulated. This supports the finding that the expression levels of ECGs are different in BxPC3 as compared to A431 and A549. BxPC3 showed least variations and A549 showed maximum variations in gene expression as the function of radiation qualities. However, expression levels of ECGs were comparable in A431 and A549 (Figure 1A).

ANOVA analysis between the three radiation qualities at $p<0.01$ revealed one gene, the ribosomal protein $18 \mathrm{~S}$, to be differentially regulated after different radiation qualities (Figure 4). This gene is one of the most commonly used internal control genes for normalisation of qRT-PCR based gene expression data. Therefore, caution needs to be practised in using this gene as an internal control gene, in particular when radiation effects are investigated.

The results in this section corroborated the findings of many other previous studies that the ECGs might be differentially regulated depending upon the experimental set-up and the cell type [8-25]. More importantly, this analysis seems to suggest that the ECGs are differentially regulated by the different cell types and radiation qualities. We attempted to confirm this observation employing a systematic analysis of the expression levels.

\section{Identification of appropriate ECGs}

Gene expression levels obtained using PCR should be appropriately normalized by one or more carefully selected stable internal control genes. The geNorm algorithm developed by Vandesompele et al. [26] can determine the expression stability of control genes on the basis of non-normalized expression levels. This measure relies on the principle that the expression ratio of two internal control genes is constant in all samples regardless of the experimental condition or cell type. This algorithm computes a gene expression stability measure $(M)$ for each gene based on the average pairwise expression ratio and then performs a stepwise exclusion of the least 

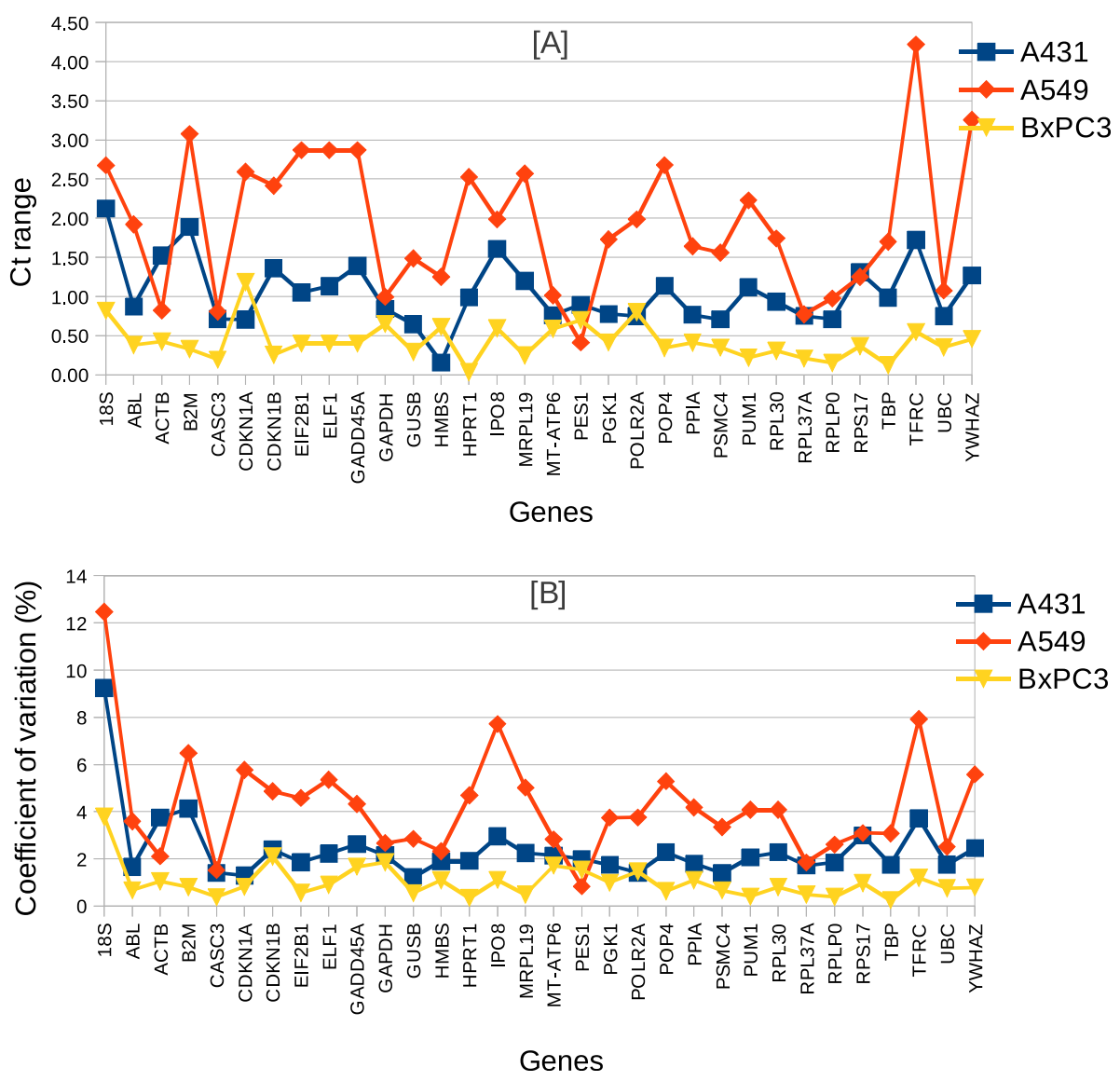

Figure 1 Radiation induced variation in ECG expression. (A) CT range and (B) coefficient of variation Figure shows the variations in the expression level of each ECG in A431, A549 and BxPC3. BxPC3 showed least variation as a function of different radiation qualities as compared to A431 and A549. A549 showed highest variations.

stable gene. Then the $M$ values are computed again and stepwise exclusion performed until two genes are left. The genes with the lowest $M$ values are considered to be the most stable across all the samples for each cell line.

\section{Ranking of the 32 ECGs}

The $M$ values for all the 32 ECGs in A431, A549 and BxPC3 computed using the geNorm algorithm (integrated into SUMO software) were sorted and ranked in Table 2. This table revealed that the two most stable ECGs irrespective of the radiation qualities were: $P G K 1$ RPL37A for A431, RPLPO-UBC for A549, and RPL37ARPLPO for BxPC3.

The actual stepwise exclusion of the worst-scoring ECG was displayed in Figure 5. In this figure: (1) there was a very steep decrease in the average $M$ value for A431 pointing at two unstably expressed ECGs, (2) the irregular decrease in the average $M$ value for A549 showed that it has aberrantly expressed ECGs, (3) the regular decrease in the average $M$ value for BxPC3 might mean that all the ECGs were stable.

\section{Calculation of normalization factor}

For each cell line, the normalization factors $(N F)$ were computed, first for the three most stable ECGs, by taking the geometric mean of their expression levels. This is followed by stepwise inclusion of the most stable remaining ECG. Then the pairwise variations $V_{n(n+1)}$ were calculated for every series of $N F_{n}$ and $N F_{n+1}$, reflecting the effect of adding an $(n+1)$ th ECG (Figure 6) [26].

Figure 6 shows that the value of $V_{3 / 4}$ was low for A431, implying that the first 3 ECGs (PGK1, RPL37A, PSMC4) were sufficient to be used for normalization. For A549, the low value of $V_{6 / 7}$ indicated that the first 6 ECGs (RPLPO, UBC, GAPDH, MT-ATP6, CASC3, PES1) 


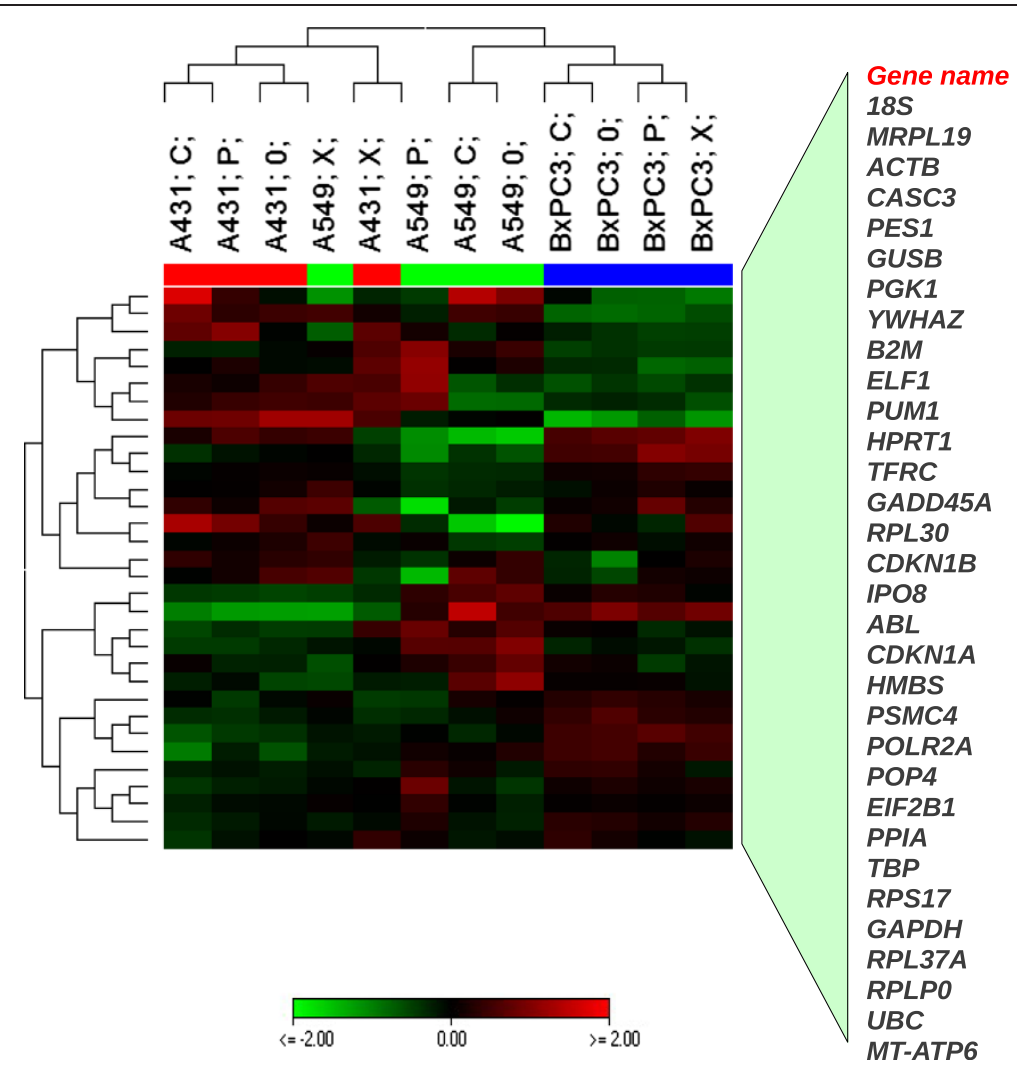

Figure 2 Direct clustering of 32 ECGs expression. This Heat map represents expression of all 32 ECGs across the three cell lines (A431, A549 and BxPC3) irradiated with photon, proton and carbon ion. Genes were hierarchically clustered by Pearson correlation coefficient using average linkage. Green denotes genes with relatively decreased expression while red denotes genes with relatively increased expression. Scale bar represent $\log _{2}$ expression level of ECGs. $C=$ Carbon, $P=$ Proton, $X=$ Photon and $0=$ Control. Expression profile of A431 and A549 are similar as compared to BxPC3 which showed unique expression profile with less variations among the samples.

were sufficient for normalization. In BxPC3, the three most stable ECGs (RPL37A, RPLPO, CASC3) were sufficient for normalization purposes.

\section{Validation of the gene-stability measure $M$}

According to Vandesompele et al. [26], three different normalization factors were calculated based on the geometric mean of three genes with, respectively, the smallest $M$ value $\left(N F_{3(1-3)}\right)$, the intermediate $M$ value $\left(N F_{3(11-13)}\right)$ and the highest $M$ value $\left(N F_{3(30-32)}\right)$ as determined by geNorm (Table 2). Further, we determined the average gene-specific variation of the three genes with the most stable expression (i.e., the smallest coefficient of variation) for each normalization factor within each cell line (Figure 7).It is conceivable that the gene-specific variation in all the cell lines were the least when the data are normalized to $\left(N F_{3(1-3)}\right)$. This validated that the gene-stability measure effectively identified the ECGs with the most stable expression.

\section{Radiation-specific expression of the ECGs within the cell lines}

The gene stability measure $M$ value was determined and validated, the candidate ECGs within each cell line were normalized by the appropriate stable ECGs found above and plotted in Figure 8. The variation in regulation of $18 \mathrm{~S}$ indicated that its expression depended upon the radiation quality (Figure 8A). This observation is in line with other above mentioned analysis performed (Figures 2 and 4). In addition, GADD45A was differentially regulated by radiotherapy in all three cell lines (Figure 8). Together, these data confirm differential regulation of candidate control genes as a function of different radiation qualities.

\section{Identifying the consensus set of ECGs for comparative studies across all cell types}

To compare the ECGs expression levels across A431, A549 and BxPC3 cells, first a consensus set of ECGs was 


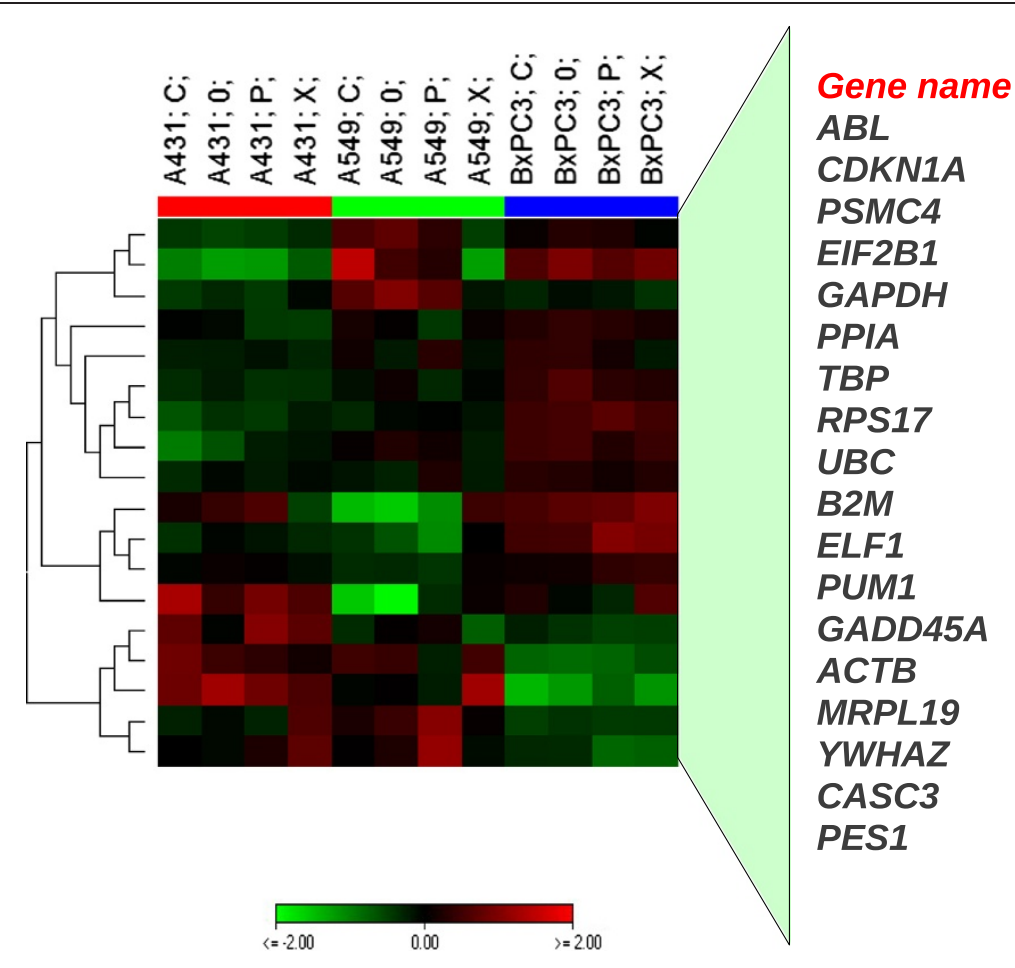

Figure 3 Cell type specific regulation of ECGs. This Heat map represents 18 ECGs which are significantly regulated according to ANOVA between the three cell lines, $p<0.05$. Relative expression of each gene were normalized to the average intensity of the gene over entire samples (virtual pool). Green denotes genes with relatively decreased expression while red denotes genes with relatively increased expression. Genes are hierarchically clustered by Pearson correlation coefficient using average linkage. Scale bar represent $\log _{2}$ expression level of ECGs. C=Carbon, $\mathrm{P}=$ Proton, $\mathrm{X}=$ Photon and $0=$ Control. Expression levels of the ECGs in BxPC3 is different as compared to A431 and A549. A549 showed maximum variation among the samples.

identified for normalization of expression data using the algorithm suggested by Vandesompele et al. [26]. RPLPO, $U B C, P P I A, T B P$ and PSMC4 were selected by eliminating the ECGs with high $M$ value to normalize and compare the cell type specific gene-expressions (Figure 9). Although the overall abundance of most ECGs among different cell lines was relatively similar, cell-line specific gene-expression were identified for some candidate ECGs such as, 18S, B2M, YWHAZ, PGK1, CDKN1A and GADD45A. In contrast, ECGs with a relatively constant expression included GAPDH, RPLPO, RPL30A, PPIA, UBC etc. In A431, a 422fold expression difference was observed between the most stable gene (PGK1) and the least stable gene (18S) whereas a 530 and 375 fold difference in expression was found in A549 and BxPC3, respectively.

\section{Discussion}

The emergence of a growing number of particle therapy facilities worldwide will stimulate comparative studies aiming to decipher the molecular mechanisms underlying differential biological effects of these novel radiation qualities.
Comparative investigation of gene regulation on transcriptional level as the function of radiation treatment constitutes a cornerstone of these studies. Quantitative real time PCR (qRT-PCR) is considered the most sensitive method for detection of gene expression level. One limitation of this method is the need for proper endogenous control gene. To generate relative expression levels, the expression of the reference gene/s needs minimally alter among different types of cells or treatments. The goal of this study was to identify such ECGs.

We analysed the expression levels of 32 ECGs using the clustering, statistical methods such as ANOVA and the geNorm algorithm. Global analysis lead to the finding that gene expression profile in pancreatic cancer cells (BxPC3) is different as compared to the two other epithelial cancer cells tested i.e. epidermoid and lung carcinoma cells (A431 and A549). The ECGs in BxPC3 showed least variation in expression whereas A549 showed maximum variation in expression as the function of radiation qualities. Among the three cell lines, the ECGs were more stable in BxPC3. From the point of view of selecting appropriate 


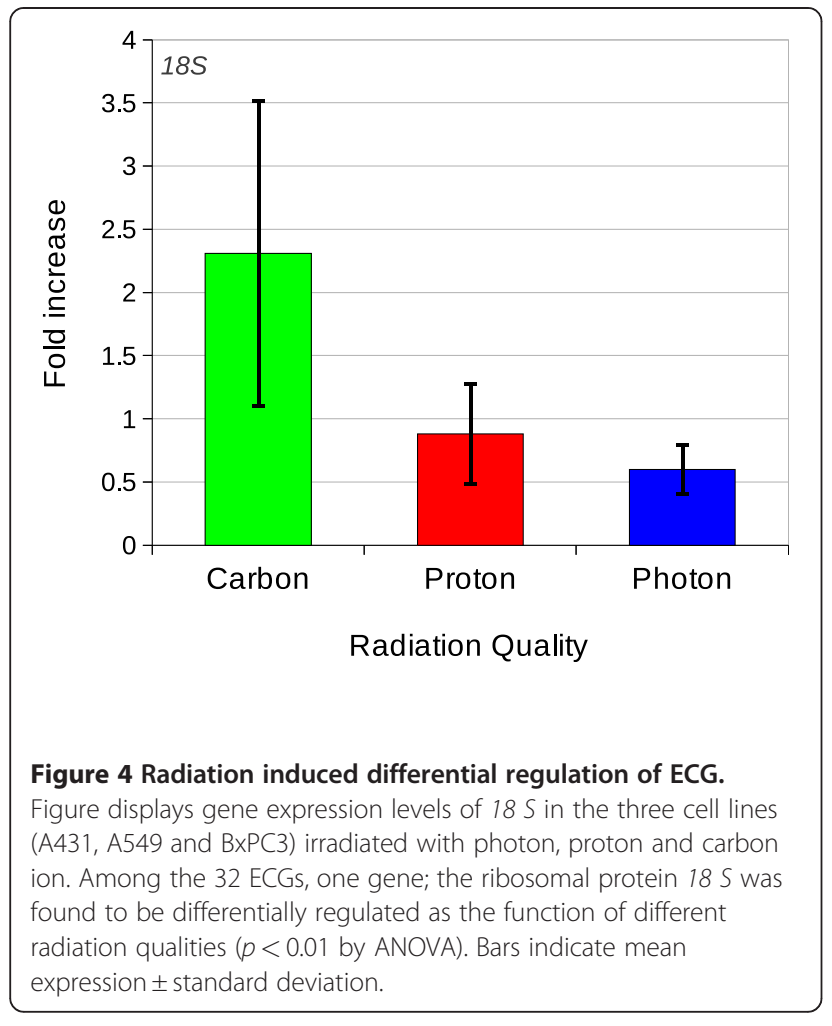

ECGs this feature might be advantageous. On the other hand, it means that the ECGs in BxPC3 are less regulated by different radiation qualities.

ANOVA analysis of non normalized data revealed that 18 out of the 32 ECGs showed cell type specific differential regulation as shown by the differences in the expression profile between A431, A549 and BxPC3. In addition, significant radiation quality specific regulation was shown by one gene the ribosomal protein $18 \mathrm{~S}$. Of note, this gene is one of the most commonly used internal control genes. Therefore, our data suggest validation of this gene prior to its use as internal control in radiation biology studies.

Although the clustering and ANOVA analysis of the raw data provided an overall overview and information about ECGs regulation, they do not allow the selection of appropriate stable ECGs for normalization of the qRT-PCR data. Next, the geNorm algorithm was employed to test for the stability of the 32 candidate ECGs as reference genes as well as selection of the optimal number of genes for normalization of gene expression.

Using the geNorm algorithm the two most stable ECGs for each cell line were determined- PGK1-RPL37A in A431, RPLPO-UBC in A549, and RPL37A-RPLPO in BxPC3. Further the optimal number of ECGs for the normalization of gene expression in each cell line was determined and validated: three ECGs each for A431 (PGK1, RPL37A, and PSMC4) and BxPC3 (RPL37A, $R P L P O$ and $C A S C 3)$ were recommended. In contrast, six
Table 2 Control genes ranked in order of their expression stability*

\begin{tabular}{|c|c|c|c|c|c|}
\hline A431 & $M$ & A549 & $M$ & BXPC3 & $M$ \\
\hline PGK1-RPL37A & 0.02 & RPLPO-UBC & 0.12 & RPL37A-RPLPO & 0.03 \\
\hline PSMC4 & 0.04 & GAPDH & 0.13 & CASC3 & 0.03 \\
\hline GUSB & 0.07 & MT-ATP6 & 0.16 & RPL30 & 0.08 \\
\hline RPLPO & 0.08 & CASC3 & 0.19 & $U B C$ & 0.08 \\
\hline$U B C$ & 0.09 & PES1 & 0.27 & RPS17 & 0.08 \\
\hline TBP & 0.12 & RPS17 & 0.31 & $E \mid F 2 B 1$ & 0.10 \\
\hline GAPDH & 0.17 & $A C T B$ & 0.32 & $A C T B$ & 0.12 \\
\hline PPIA & 0.16 & TBP & 0.42 & POP4 & 0.13 \\
\hline$A B L$ & 0.16 & PPIA & 0.39 & PSMC4 & 0.13 \\
\hline HPRT1 & 0.17 & HMBS & 0.41 & PPIA & 0.13 \\
\hline RPL30 & 0.17 & PSMC4 & 0.46 & $A B L$ & 0.14 \\
\hline PUM1 & 0.19 & $A B L$ & 0.51 & PGK1 & 0.14 \\
\hline ELF1 & 0.20 & POLRZA & 0.54 & $T B P$ & 0.14 \\
\hline POLRZA & 0.22 & PUM1 & 0.60 & GUSB & 0.15 \\
\hline CDKN1A & 0.23 & RPL30 & 0.64 & MRPL19 & 0.17 \\
\hline POP4 & 0.28 & $E I F 2 B 1$ & 0.65 & CDKN1A & 0.17 \\
\hline PES1 & 0.32 & HPRT1 & 0.66 & HPRT1 & 0.18 \\
\hline MT-ATP6 & 0.33 & CDKN1B & 0.67 & PUM1 & 0.20 \\
\hline CASC3 & 0.32 & MRPL19 & 0.65 & HMBS & 0.21 \\
\hline RPS17 & 0.33 & RPL37A & 0.68 & MT-ATP6 & 0.21 \\
\hline HMBS & 0.34 & ELF1 & 0.73 & $B 2 M$ & 0.23 \\
\hline$E \mid F 2 B 1$ & 0.37 & GUSB & 0.87 & GAPDH & 0.25 \\
\hline YWHAZ & 0.39 & YWHAZ & 0.88 & PES1 & 0.27 \\
\hline CDKN1B & 0.40 & POP4 & 0.92 & POLR2A & 0.27 \\
\hline MRPL19 & 0.43 & PGK1 & 0.97 & YWHAZ & 0.32 \\
\hline ACTB & 0.48 & GADD45A & 1.04 & ELF1 & 0.33 \\
\hline GADD45A & 0.51 & $B 2 M$ & 1.01 & TFRC & 0.36 \\
\hline IPO8 & 0.52 & TFRC & 1.22 & IPO8 & 0.37 \\
\hline$B 2 M$ & 0.52 & CDKN1A & 1.28 & 185 & 0.41 \\
\hline TFRC & 0.64 & 185 & 1.27 & GADD45A & 0.45 \\
\hline 185 & 0.96 & IPO8 & 1.34 & CDKN1B & 0.58 \\
\hline
\end{tabular}

${ }^{*} M$ values increasing from top to bottom; the two most stable control genes in each cell type, for example $R P L P O$ and $U B C$ in $A 549$, cannot be ranked in order because of the required use of gene ratios for gene-stability measurements.

ECGs for A549 data (RPLPO, UBC, GAPDH, MTATP6, $C A S C 3$ and PES1) were required.

The normalized gene expression for each cell line in Figure 8 showed that $18 S$ and $B 2 M$ are unstable genes under different radiation qualities. This is in contrast to earlier studies using $18 S$ and $B 2 M$ as reference genes for proton therapy [4]. Besides, PPIA, ACTB and $U B C$ for particle therapy using 0.5 Gy $4 \mathrm{He}$ ions in normal human lung fibroblasts [27] and $A C T B$ for A549 [5] were reported as reference genes. However, Table 2 showed 

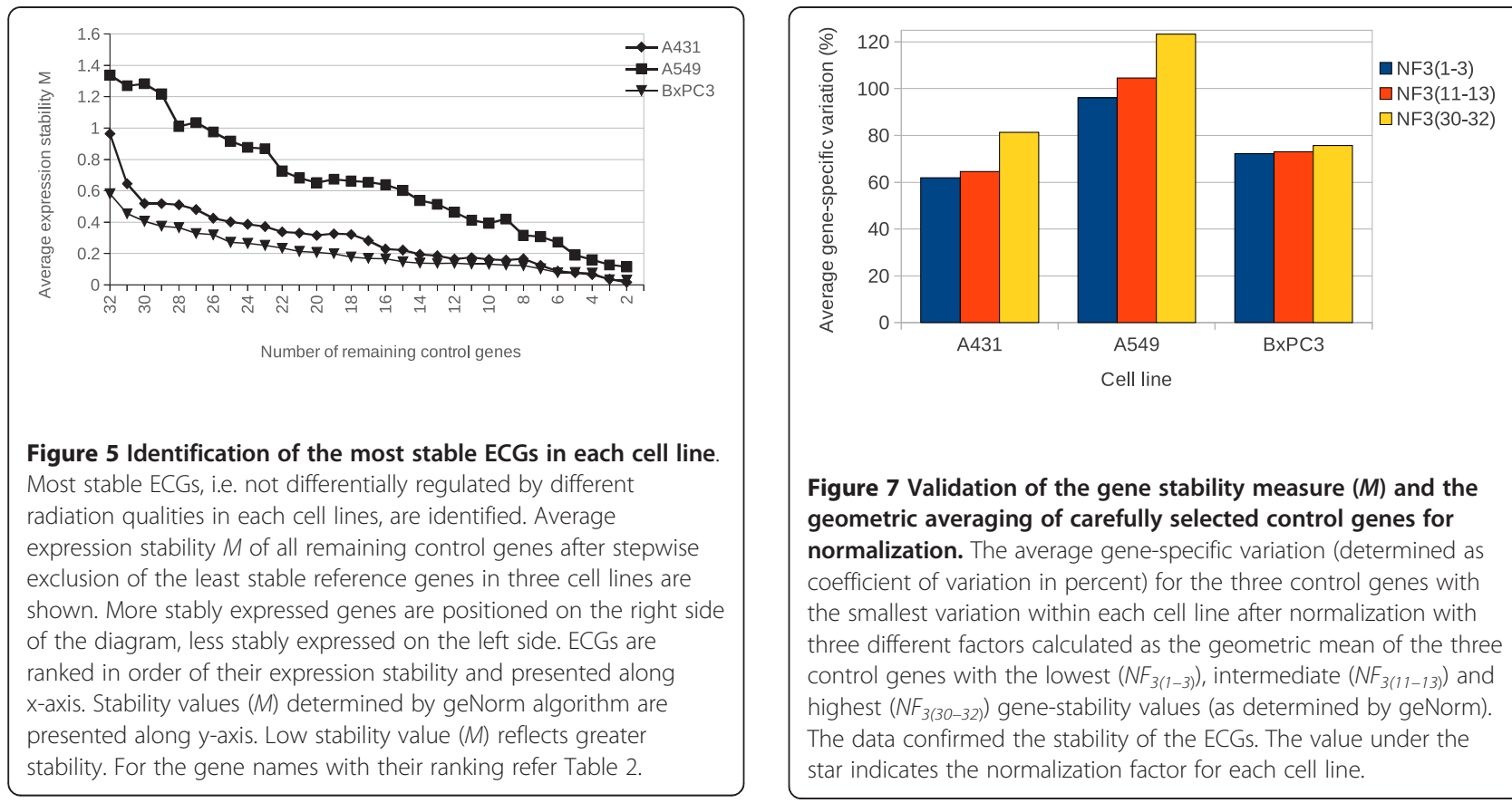

Figure 7 Validation of the gene stability measure $(M)$ and the geometric averaging of carefully selected control genes for normalization. The average gene-specific variation (determined as coefficient of variation in percent) for the three control genes with the smallest variation within each cell line after normalization with three different factors calculated as the geometric mean of the three control genes with the lowest $\left(N F_{3(1-3)}\right)$, intermediate $\left(N F_{3(11-13)}\right)$ and highest $\left(N F_{3(30-32)}\right)$ gene-stability values (as determined by geNorm). The data confirmed the stability of the ECGs. The value under the star indicates the normalization factor for each cell line.

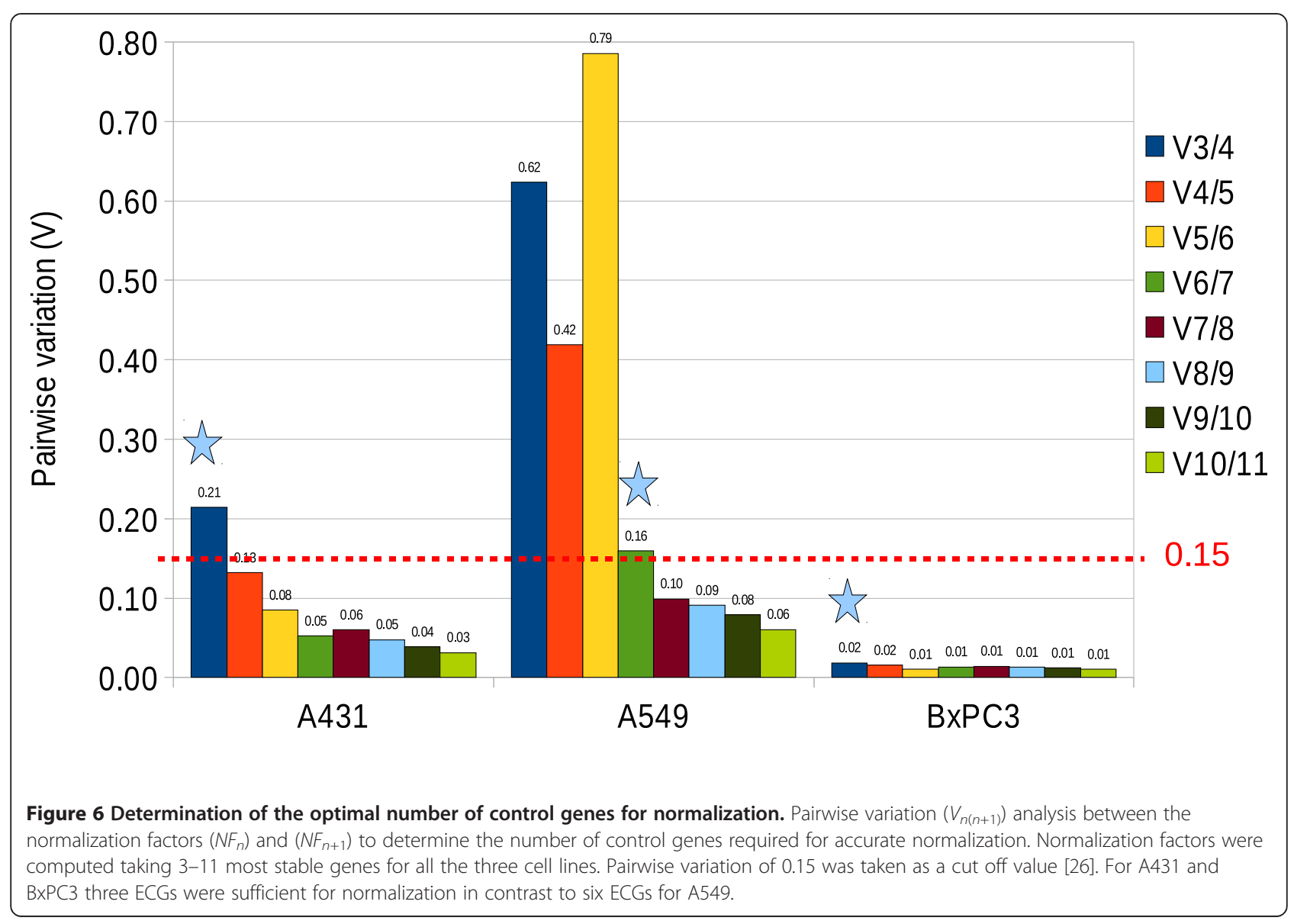




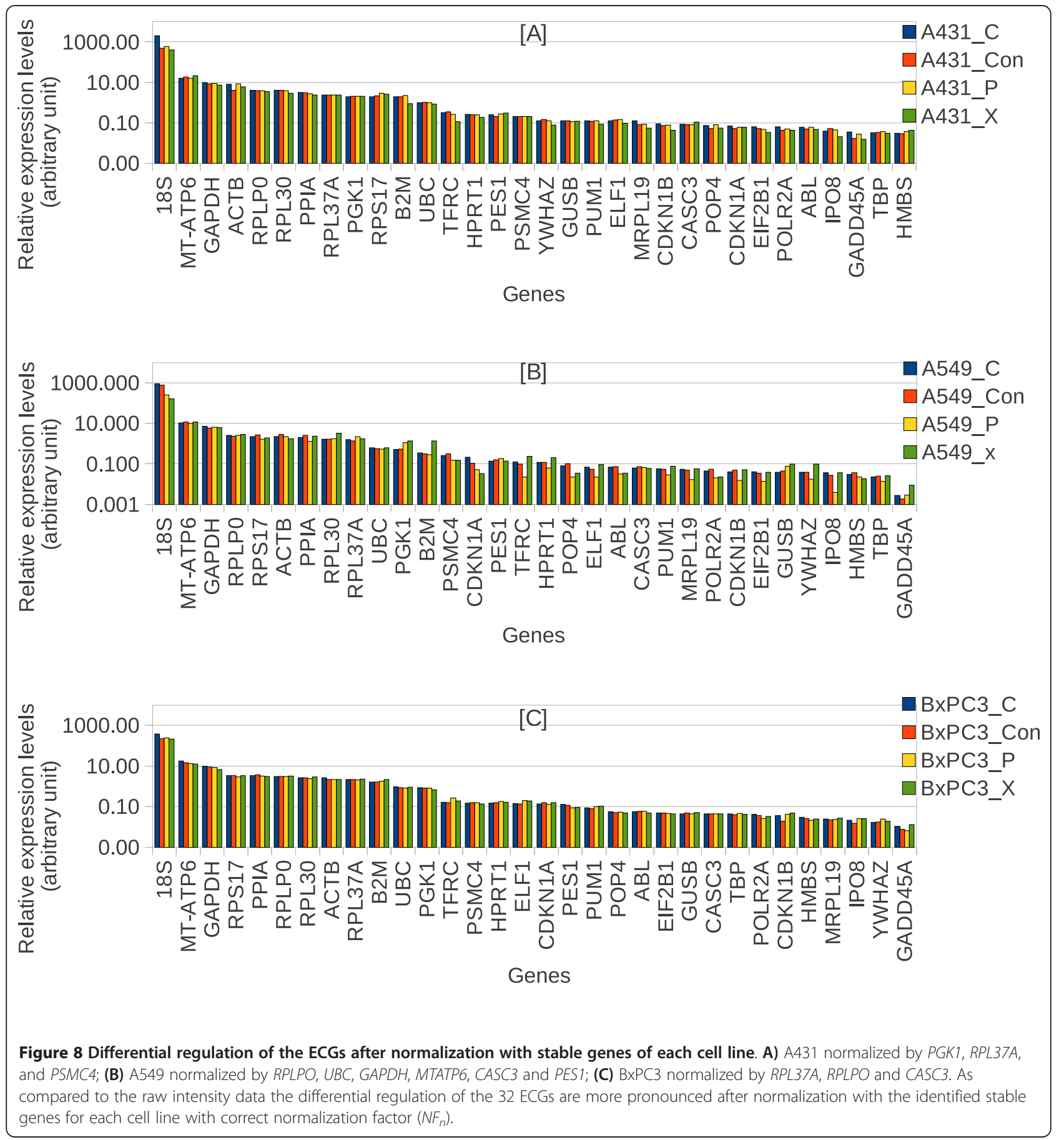

that these genes have intermediate stability within the cell line examined here.

Figure 8 demonstrates the regulation of particular genes treated with different radiation qualities. For instance, Figure 8B showed that in A549, CDKN1A- a gene downstream of p53 pathway which is also implicated in regulation of cell growth and cell response to DNA damage-- is up regulated under all the radiation qualities, with maximum up regulation under photon.
The gene YWHAZ- involved in signal transduction by binding to phosphorylated serine residues on a variety of signaling molecule-- is up regulated in A431- photon while minimally regulated in A549-photon. GADD45Awhich binds to proliferating cell nuclear antigen, stimulates DNA excision repair in vitro and inhibits entry of cells into S phase-- was down regulated in A431 but up regulated in BxPC3. 18S- a component of the ribosome, the protein manufacturing machinery of all living cells-- 


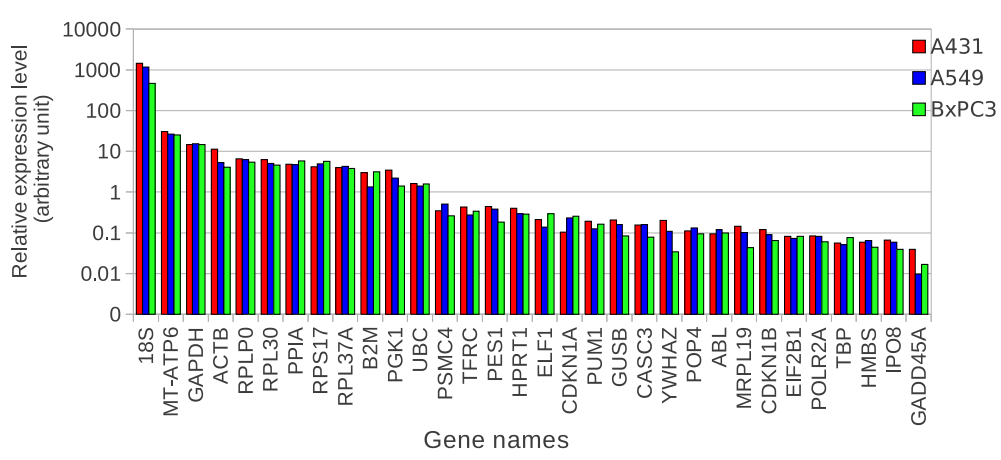

Figure 9 Logarithmic histogram of the expression levels of 32 ECGs in all the cell lines. The 32 ECGs were normalized to the geometric mean of five control genes (RPLPO, UBC, TBP, PPIA and PSMC4). In A431, a 422-fold expression difference is observed between the most stable gene ( $P$ GK1) and the least stable gene (18 S), while in A549 and BXPC3, a fold difference of 530 and 375 respectively were observed between them. In addition it shows the cell line specific differences in expression levels of particular genes (e.g. YWHAZ).

is seen to be up regulated in carbon ion while its regulation varies for proton and photon in different cell lines.

In addition, five most stable ECGs (RPLPO, $U B C, T B P$, PPIA and PSMC4) in three cell lines were selected as internal control genes for the normalisation of the gene expression independent of radiation qualities and cell type. This selection was based on the guideline of Vandesompele et al. [26].

The gene expression in each cell lines normalized by the selected five stable ECGs was shown in Figure 9. The expression of $A C T B$ showed 2.8-fold difference between the highest and lowest expression levels, whereas YWHAZ, 18S, GADD45A showed 5.8, 3.14 and 4-fold difference between the highest and lowest expression levels. The expression Heat map (Figure 3) also illustrate the regulation of YWHAZ, GADD45A and $18 S$.

Selecting the ECGs for normalization across all the cell lines is a subtle issue. Although in [26], an algorithm to select the best ECGs within each specific cell line is presented, a clear method of selecting the best ECGs for all the cell lines is not given. More precisely, among the 5 selected ECGs (Table 3) -- TBP, UBC, RPLPO, PPIA, PSMC4-- one ECG could be stable in one cell line, while it could be of intermediate stability in the other. However, our selection is supported by the fact that, in Table 2, all these genes are of intermediate stability in each of the cell lines.

\section{Conclusions}

Careful selection and validation of ECGs prior to conducting radiation biology experiment is warranted. We report that different radiation qualities induced differential regulation of a number of ECGs among the candidate 32 "housekeeping genes". Additional cell type specific gene expression was observed. Identification of the best internal control gene is a prerequisite for a successful quantitative measure of gene expression via RTPCR. In this paper we provide a template for the identification of appropriate ECGs for the radiation induced gene expression studies. We identified reliable genes for individual expression profiling of the cell lines, the normalization of A431 may be done by PGK1, RPL37A and PSMC4; A549 by RPLPO, UBC, GAPDH, MT-ATP6, CASC3 and PES1; and BxPC3 by RPL37A, RPLPO and CASC3. However, the 5 ECGs-- TBP, UBC, RPLPO, PPIA, PSMC4 - can be taken as the most suitable candidate reference genes for radiation response expression profiling in the tumor models studied. Moreover, this robust set of the most suitable candidate ECGs for radiation experiment may be applied and validated for the clinicopathological analysis of cancer specimens of epithelial tumors, non-small cell lung cancer and pancreatic adenocarcinoma.

\section{Methods \\ Cell lines}

The three different human tumour cell lines, i.e., the lung carcinoma cells (A549), the epidermoid carcinoma cells

Table 3 List of ECGs qualified as internal control genes across the cell and radiation qualities

\begin{tabular}{ll}
\hline Symbol & Name \\
\hline RPLPO & Ribosomal protein, large, P0 \\
UBC & Ubiquitin $C$ \\
PSMC4 & Protease 265 subunit, ATPase, 4 \\
PPIA & Peptidylprolyl isomerase A \\
TBP & TATA box binding protein \\
\hline
\end{tabular}


(A431) and pancreatic cancer cells (BxPC3) were used for the study. A549 and A431 cell lines were obtained from Deutsche Sammlung von Mikroorganismen und Zellkulturen GmbH (DSMZ) and BxPC3 from the American Type Culture Collection. The A549 and A431 cell lines were grown in $5 \mathrm{ml}$ Dulbeccos Modified Eagle's Medium (DMEM) (Biochrom), BxPC3 was grown in $5 \mathrm{ml}$ RPMI 1640 medium (GIBCO Invitrogen) supplemented with 10.0\% FCS in T25 flasks (Becton Dickinson). Cells were cultured under standard conditions in a fully humidified incubator with $5.0 \% \mathrm{CO}_{2}$ at $37.0^{\circ} \mathrm{C}$.

\section{Irradiation}

Cells were irradiated in T25 flasks with 2Gy of photon, $2 \mathrm{~Gy}$ of proton and 1Gy of carbon ion. Photon was delivered by a linear accelerator at $6 \mathrm{Mev}$ (Mevatron, Siemens, Erlangen, Germany). Particle irradiation with proton and carbon ion was done using a pencil beam in a spread out Bragg peak with $1.5 \mathrm{~cm}$ width equivalent to a depth of $14.0 \mathrm{~cm}$ in water, at the Heidelberg Ion Therapy Center (HIT) [28]. After irradiation, the cells were incubated for $12 \mathrm{~h}$ at $37.0^{\circ} \mathrm{C}$. Control cells were treated identically but without irradiation. Cells were scrapped using the cell scraper after adding $300.0 \mu \mathrm{l}$ TRIzol (Invitrogen) and collected in $1.5 \mathrm{ml}$ Eppendorf tubes and subsequently stored at $-20.0^{\circ} \mathrm{C}$.

\section{RNA isolation and CDNA synthesis}

RNA was isolated in phase lock tubes using TRIzol (Invitrogen) according to the manufacturer's protocol. To avoid genomic DNA contamination RNA was treated with Dnase I (Ambion). Purified RNA was eluted in $20.0 \mu \mathrm{L}$ of nuclease-free water and stored at $-20.0^{\circ} \mathrm{C}$. RNA concentration and purity was assessed using a Nanodrop ND-1000 spectrophotometer (Peqlab). Integrity and concentration of RNA samples were determined by using RNA 6000 Nano Lab Chip kits and a 2100 Bioanalyzer (Agilent). RNA $(2.0 \mu \mathrm{g})$ was subjected to reverse transcription reaction using the high-capacity cDNA reverse transcription kit (Applied Biosystems) according to the manufacturer's protocol.

\section{Real-time PCR}

To assess the expression of Human Endogenous Control gene set, real-time quantitative reverse transcription PCR (qRT-PCR) was performed on 32 candidate genes using TaqMan ${ }^{\circledR}$ chemistry (Applied Biosystems). Experiments were performed at least in triplicates for the three cell lines-- A431, A549 and BxPC3. Plates were run on a 7900HT Fast Real-Time PCR Systems (Applied Biosystems) using Fast 96-well blocks, Standard Fast PCR cycling protocol with $10.0 \mu \mathrm{L}$ reaction volumes. Cycling conditions used were-- 1 cycle initiation at $50.0^{\circ} \mathrm{C}$ for $2 \mathrm{~min}$ and 1 cycle at $95.0^{\circ} \mathrm{C}$ for $10 \mathrm{~min}$, followed by amplification for 40 cycles at $95.0^{\circ} \mathrm{C}$ for $15 \mathrm{~s}$ and $60.0^{\circ} \mathrm{C}$ for $1 \mathrm{~min}$. Amplification data were collected via Sequence Detection Systems 2.3 software (Applied Biosystems). The CT-values were computed with RQ Manager 2.xx (Applied Biosystems).

\section{Statistical analyses}

Statistical analysis of data was performed using SUMO software package (http://www.oncoexpress.de/software/ sumo). ANOVA was used to detect variation in the expression of the ECGs across the samples according to the radiation qualities and cell lines respectively. The average expression stability measure values $(M)$ were computed using the geNorm algorithm suggested by Vandesompele et al. [26] (also incorporated in the SUMO program package).

\section{Additional files}

Additional file 1: List of 32 endogenous control genes used in the study.

Additional file 2: Cycle threshold range and coefficient of variation (CV) of 32 ECGs in each cell line. The genes are sorted by the coefficient of variation increasing from top to bottom. Four replicates were used in all the three cell lines.

\section{Competing interests}

The authors declare that they have no competing interests.

\section{Acknowledgements}

We thank Claudia Rittmüller, Christiane Rutenberg and Barbara Schwager for the excellent technical assistance. This work was supported in part by the German Krebshilfe (Deutsche Krebshilfe, Max-Eder 108876), DFG National Priority Research Program: the Tumour-Vessel Interface "SPP1190", NASA Specialized Center of Research NNJ04HJ12G, and the German Federal Ministry of Research and Technology (Bundesministerium für Bildung und Forschung - BMBF 03NUK004C).

\section{Author details}

${ }^{1}$ Molecular RadioOncology [E210], National Center for Tumor Disease (NCT), German Cancer Research Center (DKFZ), Im Neuenheimer Feld 460, 69120, Heidelberg, Germany. ${ }^{2}$ Heidelberg Ion Therapy Center (HIT), Heidelberg Institute of Radiation Oncology (HIRO), Department of Radiation Oncology, University of Heidelberg Medical School, Im Neuenheimer Feld 450, 69120, Heidelberg, Germany. ${ }^{3}$ Center of Cancer Systems Biology, NASA Specialized Center of Research, St. Elizabeth's Medical Center, Tufts University, 736 Cambridge Street [CBR 1], 02135, Boston, MA, USA.

\section{Authors contributions}

GDS and AA designed the experiment, performed research, analysed data and wrote the manuscript. CS developed software and performed data analysis and statistics. SC, LH and JD analysed data and edited the manuscript. SB and TH performed the heavy ion irradiation planning and treatment. All authors read and approved the final manuscript.

Received: 1 December 2011 Accepted: 17 May 2012

Published: 17 May 2012

\section{References}

1. Abdollahi A, Folkman J: Evading tumour evasion: current concepts and perspectives of anti-angiogenic cancer therapy. Drug Resist Updat 2010, 13:16-28.

2. Abdollahi A, Li M, Ping G, Plathow C, Domhan S, Kiessling F, Lee LB, McMahon G, Groene HJ, Lipson KE, Huber PE: Inhibition of platelet-derived 
growth factor signaling attenuates pulmonary fibrosis. J Exp Med 2005, 201:925-935.

3. Abdollahi A, Schwager C, Kleef J, Esposito I, Domhan S, Peschke P, Hauser K, Hahnfeldt P, Hlatky L, Debus J, Peters JM, Friess H, Folkmann JM and Huber PE: Transcriptional network governing the angiogenetic switch in human pancreatic cancer. Proc Natl Acad Sci 2007, doi: 10.1073/pnas.0705505104

4. Suzuki Y, Nakano T, Ohno T, Oka K: Comparison of the radiobiological effect of carbon ion beam therapy and conventional radiation therapy on cervical cancer. J Radiat Res 2007, 49:473-479.

5. Akino $Y$, Teshima T, Kihara A, Kodera-Suzumoto $Y$, Inaoka M, Higashiyama S, Furusawa Y, Matsuura N: Carbon-ion beam irradiation effectively suppresses migration and invasion of human non small-cell lung cancer cells. Int J Radiat Oncol 2009, 75:475-481.

6. Domhan S, Muschal S, Schwager C, Morath C, Wirkner U, Ansorge W, Maercker C, Zeier M, Huber PE, Abdollahi A: Molecular mechanisms of the antiangiogenic and antitumor effects of mycophenolic acid. Mol Cancer Ther 2008, 7:1656-1668.

7. Almog N, Ma L, Raychowdhury R, Schwager C, Erber R, Short S, Hlatky L, Vajkoczy P, Huber PE, Folkman J, Abdollahi A: Transcriptional switch of dormant tumors to fast-growing angiogenic phenotype. Cancer Res 2009, 69:836-844.

8. Dheda K, Huggett JF, Bustin SA, Johnson MA, Rook G, Zumla A: Validation of housekeeping genes for normalizing RNA expression in real-time PCR. Biotechniques 2004, 37:112-119.

9. Haberhausen G, Pinsl J, Kuhn C, Markert-Hahn C: Comparative study of different standardization concepts in quantitative competitive reverse transcription-PCR assays. J Clin Microbiol 1998, 36:628-633.

10. Hamalainen H, Tubman J, Vikman S, Kyrola T, Ylikoski E, Warrington JA, Lahesmaa R: Identification and validation of endogenous reference genes for expression profiling of $\mathrm{T}$ helper cell differentiation by quantitative real-time RT-PCR. Anal Biochem 2001, 299:63-70.

11. Huggett J, Dheda K, Bustin S, Zumla A: Real-time RT-PCR normalisation; strategies and considerations. Genes Immun 2005, 6:279-284.

12. Mahoney D, Carey K, Fu M, Snow R, Cameron-Smith D, Gianni Parise G, Tarnopolsky MA: Real-time RT-PCR analysis of housekeeping genes in human skeletal muscle following acute exercise. Physiol Genomics 2004, 18:226-231.

13. Barnard G, Staniunas R, Bao S, Mafune K, Steele GD Jr, John JL, Gollan JL, Chen LB: Increased expression of human ribosomal phosphoprotein PO messenger RNA in hepatocellular carcinoma and colon carcinoma. Cancer Res 1992, 52:3067-3072.

14. Henry J, Coggin D, King C: High-level expression of the ribosomal protein L19 in human breast tumors that overexpress erbB-2. Cancer Res 1993, 53:1403-1408

15. Suzuki T, Higgins P, Crawford D: Control selection for RNA quantitation. Biotechniques 2000, 29:332-337.

16. Murphy R, Watt K, Cameron-Smith D, Gibbons CJ, Snow RJ: Effects of creatine supplementation on housekeeping genes in human skeletal muscle using real-time RT- PCR. Physiol Genomics 2003, 12:163-174.

17. Ross D, Scherf U, Eisen M, Perou CM, Rees C, Spellman P, lyer V, Jeffrey $S$, de Rijn MV, Waltham M, Pergamenschikov A, Jeffrey CF, Lee JC, Lashkari D, Shalon D, Myers TG, Weinstein JN, Botstein D, Brown PO: Systematic variation in gene expression patterns in human cancer cell lines. Nat Genet 2000, 24:227-235.

18. Schmidt U, Fuessel S, Koch R, Baretton GB, Lohse A, Tomasetti S, Unversucht S, Froehner M, Wirth MP, Meye A: Quantitative multi-gene expression profiling of primary prostate cancer. Prostate 2006, 66:1521-1534.

19. Khimani A, Mhashilkar A, Mikulskis A, O'Malley M, Liao J, Golenko EE, Mayer P, Chada S, Killian JB, Lott ST: Housekeeping genes in cancer: normalization of array data. Biotechniques 2005, 38:739-745.

20. Thellin O, Zorzi W, Lakaye B, De Borman B, Coumans B, Hennen G, Grisar T, Igout $A$, Heinen $E$ : Housekeeping genes as internal standards: use and limits. Biotechnol 1999, 75:291-295.

21. Tricarico C, Pinzani P, Bianchi S, Paglierani M, Distante V, Pazzagli M, Bustin SA, Orlando C: Quantitative real-time reverse transcription polymerase chain reaction: normalization to rRNA or single housekeeping genes is inappropriate for human tissue biopsies. Anal Biochem 2002, 309:293-300.

22. Tsuji N, Kamagata C, Furuya M, Kobayashi D, Yagihashi A, Morita T, Horita S, Watanabe N: Selection of an internal control gene for quantitation of mRNA in colonic tissues. Anticancer Res 2002, 22:4173-4178.
23. Vaarala M, Porvari KS, Kyllonen AP, Mustonen MV, Lukkarinen O, Vihko PT: Several genes encoding ribosomal proteins are over-expressed in prostate-cancer cell lines: confirmation of L7a and L37 over-expression in prostate-cancer tissue samples. Int J Cancer 1998, 78:27-32.

24. Viraj P, Melissa AH, Philippa H, Hillyer P, Navarro MB, Rabin RL: Systematic method for determining an ideal housekeeping gene for real-time PCR analysis. J Biomol Tech 2008, 19:342-347.

25. Akerman G, Rosenzweig B, Domon O, Tsai C, Bishop M, McGarrity LJ, James T: Alterations in gene expression profiles and the DNA-damage response in ionizing radiation-exposed TK6 cells. Environ Mol Mutagen 2005, 45:188-205.

26. Vandesompele J, De Preter K, Pattyn F, Poppe B, Roy NV, Paepe AD, Speleman F: Accurate normalization of real-time quantitative RT-PCR data by geometric averaging of multiple internal control genes. Genome Biol 2002, 3: research0034.1-0034.11.

27. Ghandhi SA, Yaghoubian B, Amundson SA: Global gene expression analyses of bystander and alpha particle irradiated normal human lung fibroblasts: Synchronous and differential responses. BMC Medical Genomics 2008 1:63 doi:10.1186/1755-8794-1-63

28. Combs S, Jakel O, Haberer T, Debus J: Particle therapy at the Heidelberg Ion Therapy Center (HIT) - Integrated research-driven university-hospitalbased radiation oncology service in Heidelberg, Germany. Radiother Oncol 2010, 95:41-44.

doi:10.1186/1748-717X-7-70

Cite this article as: Sharungbam et al:: Identification of stable endogenous control genes for transcriptional profiling of photon, proton and carbon-ion irradiated cells. Radiation Oncology 2012 7:70.

\section{Submit your next manuscript to BioMed Central and take full advantage of:}

- Convenient online submission

- Thorough peer review

- No space constraints or color figure charges

- Immediate publication on acceptance

- Inclusion in PubMed, CAS, Scopus and Google Scholar

- Research which is freely available for redistribution 